\title{
Une généralisation du théorème de Cobham
}

\author{
par \\ S. FABRE (Villetaneuse)
}

Nous généralisons le théorème de Cobham ([2]), en démontrant qu'une partie infinie de $\mathbb{N}$ est reconnaissable en base $k$ ( $k$ entier strictement plus grand que un) et reconnaissable dans un système de numération associé à un nombre de Pisot unitaire (ayant une propriété arithmétique supplémentaire) si et seulement si elle est ultimement périodique.

\section{A. Rappels et notations}

1. Notations. Nous ne travaillons qu'avec des alphabets finis $A=\{0,1$, $\ldots, k\}$ avec $k$ un entier positif, dont les éléments $a$ sont appelés les lettres. L'ensemble $A^{*}$ designe le monoïde libre engendré par $A$ : un élément $u$ de $A^{*}$ est une suite finie de lettres de $A$ appelé un mot, le mot vide est noté par $\varepsilon$. Si pour quatre mots $u, v, s$ et $t$ de $A^{*}$ nous avons $u=v s t, v$ est un préfixe de $u, t$ un suffixe de $u$ et $s$ un facteur de $u$.

Nous notons la longueur d'un mot $u$ par $|u|$, en particulier $|\varepsilon|=0$. Le mot de longueur $n$ ne comportant que la lettre $a$ est noté par $a^{n}$.

Nous définissons sur $A^{*}$ l'ordre lexicographique (noté $\succ$ ) par :

- si $u=a_{1} \ldots a_{n}$ et $v=b_{1} \ldots b_{n}$, alors $u \succ v$ si, pour un $i \in\{1, \ldots, n\}$, $a_{i}>b_{i}$, et pour tout $j \leq i, a_{j}=b_{j}$;

- si $u=a_{1} \ldots a_{m}$ et $v=b_{1} \ldots b_{n}$ avec $m<n$, alors $u \succ v$ si $u 0^{n-m} \succ v$.

2. $\theta$-système de numération, ensemble $U_{\theta}$-reconnaissable. Un nombre de Pisot est un entier algébrique strictement plus grand que 1, dont tous les conjugués sont à l'intérieur du cercle unité. Pour $\theta$ un nombre de Pisot, soit le $\theta$-développement de 1 (noté $D_{\theta}(1)$ ) la suite infinie d'entiers positifs $\left(\alpha_{n}\right)_{n \in \mathbb{N}}$ suivante (pour plus de précision voir [9], [1]) :

- $\alpha_{0}=[\theta], r_{0}=\{\theta\}([x]$ désignant la partie entière de $x$ et $\{x\}$ la partie fractionnaire de $x)$;

- pour tout entier $n, \alpha_{n+1}=\left[\theta r_{n}\right], r_{n+1}=\left\{\theta r_{n}\right\}$. 
Le $\theta$-développement de 1 d'un nombre de Pisot est soit ultimement périodique, soit fini (i.e. $D_{\theta}(1)=\alpha_{0} \ldots \alpha_{n}\left(\alpha_{n+1} \ldots \alpha_{n+m}\right)^{\omega}$ ou $D_{\theta}(1)=$ $\left.\alpha_{0} \ldots \alpha_{n}\right)([9])$. Nous pouvons alors définir à partir de $D_{\theta}(1)$ le polynôme $Q_{\theta}(x)$ de $\mathbb{Z}[X]$ suivant $([1])$ :

- si $D_{\theta}(1)=\alpha_{0} \ldots \alpha_{n}$, alors $Q_{\theta}(x)=x^{n+1}-\alpha_{0} x^{n}-\alpha_{1} x^{n-1}-\ldots-\alpha_{n}$;

- si $D_{\theta}(1)=\alpha_{0} \ldots \alpha_{n}\left(\alpha_{n+1} \ldots \alpha_{n+m}\right)^{\omega}$, alors

$$
\begin{aligned}
Q_{\theta}(x)= & \left(x^{n+m+1}-\alpha_{0} x^{n+m}-\alpha_{1} x^{n+m-1}-\ldots-\alpha_{n+m}\right) \\
& -\left(x^{n+1}-\alpha_{0} x^{n}-\alpha_{1} x^{n-1}-\ldots-\alpha_{n}\right) .
\end{aligned}
$$

Si $\theta$ est toujours racine de $Q_{\theta}(x)$, ce polynôme peut ne pas être son polynôme minimal (noté $P_{\theta}(x)$ ). Dans toute la suite le réel $\theta$ désignera toujours un nombre de Pisot unitaire pour lequel $Q_{\theta}(x)$ est son polynôme minimal, et nous dirons que $\theta$ possède la propriété $(\mu)$.

Comme A. Bertrand ([1], mais aussi [6]), nous définissons le $\theta$-système de numération $\left(\left(U_{n}\right)_{n \in \mathbb{N}} ; A_{\theta}\right)$ de la façon suivante :

- si $D_{\theta}(1)=\alpha_{0} \ldots \alpha_{n}$, alors

$U_{0}=1 ; \quad U_{i}= \begin{cases}\alpha_{0} U_{i-1}+\alpha_{1} U_{i-2}+\ldots+\alpha_{i-1} U_{0}+1 & \text { pour } 1 \leq i \leq n \\ \alpha_{0} U_{i-1}+\alpha_{1} U_{i-2}+\ldots+\alpha_{n} U_{i-(n+1)} & \text { pour } i \geq n+1\end{cases}$

- si $D_{\theta}(1)=\alpha_{0} \ldots \alpha_{n}\left(\alpha_{n+1} \ldots \alpha_{n+m}\right)^{\omega}$, alors

$U_{0}=1 ; \quad U_{i}=\alpha_{0} U_{i-1}+\alpha_{1} U_{i-2}+\ldots+\alpha_{i-1} U_{0}+1 \quad$ pour $i \geq 1 ;$

- dans les deux cas $A_{\theta}=\{0,1, \ldots,[\theta]\}$.

Un entier $p$ admet comme représentant le mot $a_{0} \ldots a_{n}$ de $A_{\theta}^{*}$ si on a l'égalité $p=a_{0} U_{n}+\ldots+a_{n} U_{0}$. Dans ce système, tout entier non nul admet un représentant unique ne comportant aucun facteur plus grand ou égal à $D_{\theta}(1)$ pour l'ordre lexicographique ([1] et [6]), et ne débutant pas par la lettre 0 : c'est le représentant normalisé de l'entier, et nous notons par $[\theta]^{*}$ l'ensemble des représentants normalisés.

Pour la suite, $\Pi_{\theta}(x)$ (respectivement $\Pi_{k}(x)$ ) désigne la valeur numérique du mot $x$ dans le $\theta$-système de numération (respectivement en base $k$ ), et $\nu_{\theta}(n)\left(\right.$ resp. $\left.\nu_{k}(n)\right)$ le représentant normalisé de $n$ dans le $\theta$-système de numération (resp. le représentant de $n$ en base $k$ ).

EXemple 1. Nous notons $\phi=(1+\sqrt{5}) / 2$ le "nombre d'or". Nous avons $D_{\phi}(1)=11$, ce qui implique que la base du système de numération associé à $\phi$ est la suite de Fibonacci (soit 1, 2, 3, 5 etc.), et l'alphabet $A_{\phi}=\{0,1\}$. Nous trouvons le système de numération de Fibonacci ([12]) dans lequel le nombre 8 admet trois représentants ne débutant pas par un 0 : les mots 10000, 1011 et 1100, le premier étant le représentant normalisé de 8 .

Pour $\phi^{2}=(3+\sqrt{5}) / 2, D_{\phi^{2}}(1)=21^{\omega}$. Ici la base du système est la suite des entiers apparaîssant à des rangs impairs dans la suite de Fibonacci 
(soit 1, 3, 8 etc.), l'alphabet étant $A_{\phi^{2}}=\{0,1,2\}$. Le nombre 8 admet deux représentants : les mots 100 et 22 , le premier est le représentant normalisé de 8.

Dans ces deux cas $Q_{\theta}(x)$ est le polynôme minimal de $\theta$.

Soit $\nu$ le nombre de Pisot unitaire dont le polynôme minimal est $P_{\nu}(x)=$ $x^{3}-x-1$. Par calcul, nous trouvons $D_{\nu}(1)=10001$, ce qui implique que $Q_{\nu}(x)=x^{5}-x^{4}-1=P_{\nu}(x)\left(x^{2}-x+1\right)$. Nous voyons que $Q_{\nu}(1) \neq P_{\nu}(1)$, et $\nu$ sort du cadre de notre étude.

Soit $S$ une partie de $\mathbb{N}$; nous définissons le langage $L_{\theta}(S)$ comme l'ensemble des représentants normalisés des éléments de $S$ dans le $\theta$-système de numération. Par analogie avec la notion de $k$-reconnaissabilité, nous disons que la partie $S$ de $\mathbb{N}$ est $U_{\theta^{-}}$reconnaissable si $L_{\theta}(S)$ est reconnaissable par un automate ayant un nombre fini d'états (pour la théorie des automates et la $k$-reconnaissabilité nous renvoyons à [4]).

Un $\theta$-automate est un automate qui n'accepte que les mots ne comportant aucun facteur supérieur ou égal à $D_{\theta}(1)$. Par définition les $\theta$-automates ne sont pas complets dans l'alphabet sur lequel ils sont définis. Cependant, il est clair que si une partie $S$ de $\mathbb{N}$ est $U_{\theta}$-reconnaissable, $L_{\theta}(S)$ pourra être reconnu par un $\theta$-automate. De plus, cet automate pourra être choisi déterministe et minimal.

3. Substitution de longueur $\theta$, suite $\theta$-automatique. Pour nous, une substitution est un triplet $\omega=\left(\omega, A, a_{0}\right)$ où : $A$ est un alphabet fini; $\omega$ une application de $A$ dans $A^{*}$ (prolongeable en un morphisme de $A^{*}$ dans $A^{*}$ ); $a_{0}$ une lettre de $A$ telle que $\omega\left(a_{0}\right)=a_{0} u$ (avec $u$ un mot de $A^{*}$, différent du mot vide). On peut alors définir dans $A^{\mathbb{N}}$ le point fixe de la substitution, $X_{\omega}=\lim _{n \rightarrow \infty}\left(\omega\left(a_{0}\right)\right)^{n}$.

Pour $k$ entier fixé, si toutes les lettres ont pour image par $\omega$ un mot de longueur $k, \omega$ est une substitution de longueur constante $k$.

Si le mot $X_{\omega}$ est périodique (resp. ultimement périodique) la substitution $\omega$ est dite périodique (resp. ultimement périodique).

Nous rappelons brièvement quelques définitions et des résultats donnés dans [5].

- Morphisme. Soient $\omega=\left(\omega, A, a_{0}\right)$ et $\tau=\left(\tau, B, b_{0}\right)$ deux substitutions; nous disons que $h$ est un morphisme de $\omega$ dans $\tau$ si $h$ est une application de $A$ dans $B$ telle que

$$
h\left(a_{0}\right)=b_{0} ; \quad h(\omega(a))=\tau(h(a)) \quad \forall a \in A .
$$

- Conjugaison. Les substitutions $\omega=\left(\omega, A, a_{0}\right)$ et $\tau=\left(\tau, B, b_{0}\right)$ sont conjuguées s'il existe une substitution $\sigma=\left(\sigma, C, c_{0}\right)$ et deux morphismes $h_{1}$ et $h_{2}$ de $\sigma$ dans $\omega$ et de $\sigma$ dans $\tau$, respectivement. 
Propriété. $\omega$ et $\tau$ sont conjuguées si et seulement si pour tout entier $n$, $\left|\omega\left(a_{n}\right)\right|=\left|\tau\left(b_{n}\right)\right|\left(a_{n}\right.$ et $b_{n}$ désignent respectivement les $n$-ièmes lettres de $X_{\omega}$ et $\left.X_{\tau}\right)$.

- Substitution $\omega_{\theta}$. A partir de $D_{\theta}(1)$, nous définissons une substitution $\omega_{\theta}$ de la façon suivante :

- si $D_{\theta}(1)=\left(\alpha_{0} \ldots \alpha_{n}\right)$, alors $\omega_{\theta}=\left(\omega_{\theta},\{0,1, \ldots, n\}, 0\right)$ :

$$
0 \rightarrow 0^{\alpha_{0}} 1, \quad 1 \rightarrow 0^{\alpha_{1}} 2, \ldots, n-1 \rightarrow 0^{\alpha_{n-1}} n, \quad n \rightarrow 0^{\alpha_{n}} ;
$$

- si $D_{\theta}(1)=\left(\alpha_{0} \alpha_{1} \ldots \alpha_{n}\right)\left(\alpha_{n+1} \alpha_{n+2} \ldots \alpha_{n+m}\right)^{\omega}$, alors $\omega_{\theta}=\left(\omega_{\theta}\right.$, $\{0,1, \ldots, n+m\}, 0)$ :

$$
0 \rightarrow 0^{\alpha_{0}} 1, \ldots, n+m-1 \rightarrow 0^{\alpha_{n+m-1}}(n+m), \quad n+m \rightarrow 0^{\alpha_{n+m}}(n+1) .
$$

Propriétés. Le polynôme caractéristique de la matrice d'occurrences de $\omega_{\theta}$ est égal à $Q_{\theta}(x)$. La suite $\left(\left|\omega_{\theta}^{n}(0)\right|\right)_{n \in \mathbb{N}}$ est égale à la base du système de numération associé à $\theta$.

- Substitution de longueur $\theta$. Nous appelons substitution de longueur $\theta$ toute substitution conjuguée avec $\omega_{\theta}$ (définie comme précédemment).

Par extension de la définition des suites $k$-automatiques (voir par exemple [4]), nous disons que la suite $\left(b_{n}\right)_{n \in \mathbb{N}}$ appartenant à $B^{\mathbb{N}}$ ( $B$ un alphabet fini) est $\theta$-automatique si elle est obtenue par codage littéral du point fixe d'une $\theta$-substitution.

Soit $\Delta=\left\{Q, q_{0}, F,\{0, \ldots,[\theta]\}, \delta\right\}$ un $\theta$-automate; la suite des états de $\Delta$ est la suite infinie $\left[\delta\left(q_{0}, \nu_{\theta}(n)\right)\right]_{n \in \mathbb{N}}$. Nous obtenons alors un théorème et son corollaire :

THÉORÈme 0 . La suite infinie des états d'un $\theta$-automate est point fixe d'une $\theta$-substitution $\sigma=\left(\sigma, Q, q_{0}\right)$. Inversement, le point fixe d'une $\theta$ substitution est la suite des états d'un $\theta$-automate.

Corollaire 0. Une partie infinie de $\mathbb{N}$ est une partie $U_{\theta}$-reconnaissable si et seulement si sa suite caractéristique (appartenant $\grave{a}\{0,1\}^{\mathbb{N}}$ ) est $\theta$ automatique.

R e mar qu e. Le théorème 0 n'est que la version pour les $\theta$-systèmes de numération d'un théorème dû à Cobham pour le cas entier ([3]).

Nous généralisons ici le théorème de Cobham ([2], voir aussi [7] pour une autre démonstration) : "Soient $k$ et $j$ deux entiers strictement plus grands que 1 , multiplicativement indépendants (i.e. $\log k / \log j \notin \mathbb{Q}$ ). Une partie infinie de $\mathbb{N}$ est une partie $j$-reconnaissable et $k$-reconnaissable si et seulement si elle est ultimement périodique," par le théorème : "Soient $\theta$ un nombre de Pisot unitaire ayant la propriété $(\mu)$ et $k$ un entier strictement plus grand que 1 . Une partie infinie de $\mathbb{N}$ est $k$-reconnaissable et $U_{\theta}$-reconnaissable si et seulement si elle est ultimement périodique." 
Signalons d'ores et déjà que C. Frougny a montré qu'une partie ultimement périodique est $U_{\theta}$-reconnaissable ([6]).

\section{B. Indépendance entre les $k$-substitutions et les $\theta$-substitutions}

\section{Deux propositions}

Proposition 1. Soient $\theta$ un nombre de Pisot unitaire ayant la propriété $(\mu), k$ un entier strictement plus grand que 1 , et $\omega=\left(\omega, A, a_{0}\right)$ une $k$ substitution non ultimement périodique. S'il existe une lettre a de A telle que $\omega(a)$ soit le mot $a^{k}$, alors $X_{\omega}$ n'est pas $\theta$-automatique.

Preuve. Soit $\omega=\left(\omega, A, a_{0}\right)$ une $k$-substitution vérifiant les hypothèses de la proposition, et supposons que $X_{\omega}$ soit $\theta$-automatique : cela signifie qu'il existe une substitution $\sigma=\left(\sigma, B, b_{0}\right)$ de longueur $\theta$, et une application $\Phi$ de $B$ dans $A$, telles que $\Phi\left(X_{\sigma}\right)=X_{\omega}$.

Nous pouvons considérer le mot $X_{\omega}$ comme la suite infinie des états d'un $k$-automate $\Delta_{k}=\left\{A, a_{0},\{0, \ldots, k-1\}, \delta_{k}\right\}([3])$, et le mot $X_{\sigma}$ comme celle d'un $\theta$-automate $\Delta_{\theta}=\left\{B, b_{0},\{0, \ldots,[\theta]\}, \delta_{\theta}\right\}$ (théorème 0 ).

Nous avons dans $\Delta_{k}$ :

$$
\begin{gathered}
\exists u \in\{0, \ldots, k-1\}^{*} \quad \text { tel que } \quad \delta_{k}\left(a_{0}, u\right)=a ; \\
\forall v \in\{0, \ldots, k-1\}^{*}, \quad \delta_{k}(a, v)=a \quad\left(\operatorname{car} \omega(a)=a^{k}\right) .
\end{gathered}
$$

Donc

On obtient

$$
\forall v \in\{0, \ldots, k-1\}^{*}, \quad \delta_{k}\left(a_{0}, u v\right)=a .
$$

$$
\forall \alpha \in \mathbb{N} \forall n \in\left[\Pi_{k}\left(u 0^{\alpha}\right), \Pi_{k}\left(u 0^{\alpha}\right)+k^{\alpha}\left[, \quad \delta_{k}\left(a_{0}, \nu_{k}(n)\right)=a .\right.\right.
$$

Comme $X_{\omega}$ n'est pas ultimement périodique, il existe une lettre récurrente $b$ de $B$ (i.e. $b$ apparaît une infinité de fois dans $X_{\sigma}$ ) telle que $\Phi(b)$ soit différente de $a$. Cela implique que l'état $b$ est récurrent dans $\Delta_{\theta}$ : il existe $(w, s, t) \in\{0, \ldots,[\theta]\}^{*}$ et $w$ différent du mot vide tel que pour tout $\beta \in \mathbb{N}$, $\delta_{\theta}\left(b_{0}, s w^{\beta} t\right)=b$.

Nous allons obtenir une contradiction en montrant qu'il existe un couple d'entiers $(\alpha, \beta)$ tel que

$$
\Pi_{k}\left(u 0^{\alpha}\right) \leq \Pi_{\theta}\left(s w^{\beta} t\right)<\Pi_{k}\left(u 0^{\alpha}\right)+k^{\alpha} .
$$

Comme $\theta$ est un nombre de Pisot on peut voir que $\Pi_{\theta}\left(s w^{\beta} t\right)=M \theta^{\beta|w|}+$ $L+o\left(\varrho^{\beta|w|}\right)$, où $L$ et $M$ sont deux constantes réelles dépendant de $(w, s, t)$, $|\varrho|<1$, et $\lim _{\beta \rightarrow \infty} o\left(\varrho^{\beta|w|}\right)=0$. Les inégalités (a) sont alors équivalentes à

$$
\begin{aligned}
\alpha \log k+\log \left(\Pi_{k}(u)\right) & \leq \log M+\beta \log \theta^{|w|}+\log \left(1+\frac{L+o\left(\varrho^{\beta|w|}\right)}{M \theta^{\beta|w|}}\right) \\
& <\alpha \log k+\log \left(\Pi_{k}(u)+1\right) .
\end{aligned}
$$


Nous supposons $\beta$ grand pour pouvoir négliger le terme $\log (1+\ldots)$. Nous obtenons donc les inégalités suivantes :

$$
\alpha+\frac{\log \left(\Pi_{k}(u)\right)}{\log k} \leq \beta \frac{\log \theta^{|w|}}{\log k}+\frac{\log M}{\log k}<\alpha+\frac{\log \left(\Pi_{k}(u)+1\right)}{\log k} .
$$

L'entier $\beta$ recherché doit vérifier

$$
\left\{\frac{\log \left(\Pi_{k}(u)\right)-\log M}{\log k}\right\} \leq\left\{\beta \frac{\log \theta^{|w|}}{\log k}\right\}<\left\{\frac{\log \left(\Pi_{k}(u)+1\right)-\log M}{\log k}\right\} .
$$

Mais $\log \theta^{|w|} / \log k$ est irrationnel, ce qui implique que la suite $\left(n \log \theta^{|w|} / \log k\right)_{n \in \mathbb{N}}$ est équirépartie modulo 1 . Il existe donc une infinité d'entiers $\left(\beta_{j}\right)_{j \in \mathbb{N}}$ vérifiant l'inégalité précédente. Pour chaque $\beta_{j}$ on pose

$$
\alpha_{j}=\left[\beta_{j} \frac{\log \theta^{|w|}}{\log k}\right]-\left[\frac{\log \left(\Pi_{k}(u)\right)-\log M}{\log k}\right] .
$$

Le couple $\left(\alpha_{j}, \beta_{j}\right)$ vérifie alors les inégalités (a) dès que $\beta_{j}$ est assez "grand". Nous obtenons ainsi la contradiction souhaitée, ce qui démontre la proposition 1.

DÉfinition. Soient $\omega=\left(\omega, A, a_{0}\right)$ une substitution et $[0 \ldots N]$ le mot de longueur $N$ préfixe de $X_{\omega}$. Soit $E_{t}$ l'ensemble des entiers défini par $E_{t}=$ $\{0\} \cup\left\{\left|\omega^{t}([0 \ldots N])\right|, \forall N \in \mathbb{N}\right\}$. La substitution $\omega$ est reconnaissable à droite et à gauche à l'ordre $t$ s'il existe un entier $L_{t}$ (dépendant de $t$ ) tel que si $n$ appartient à $E_{t}$, et si, pour $m$ entier, les mots $\left[n-L_{t} \ldots n+L_{t}\right]$ et $\left[m-L_{t} \ldots m+L_{t}\right]$ de $X_{\omega}$ centrés en $n$ et $m$ respectivement sont identiques, alors $m$ appartient à $E_{t}$.

Dans le cas où $\omega$ est une $k$-substitution, cela implique que $m$ est congru à $n$ modulo $k^{t}$.

Si quel que soit l'entier $t, \omega$ est reconnaissable à droite et à gauche à l'ordre $t$, nous disons que $\omega$ est reconnaissable à tous les ordres.

R e marque. Cette notion a été étudiée par B. Mossé ([8]), dont nous utiliserons certains résultats. Nous renvoyons aussi à [10] pour la notion de reconnaissabilité.

Proposition 2. Soient $\theta$ un nombre de Pisot unitaire ayant la propriété $(\mu), k$ un entier strictement plus grand que 1 et $\omega=\left(\omega, A, a_{0}\right)$ une $k$-substitution reconnaissable à tous les ordres. Alors $X_{\omega}$ n'est pas $\theta$ automatique.

Avant de donner la preuve de la proposition, nous énonçons deux lemmes :

Lemme 1. Soit une $\theta$-substitution $\sigma=\left(\sigma, B, b_{0}\right)$, et $b$ une lettre de $B$. Alors, $\lim _{n \rightarrow \infty}\left|\sigma^{n}(b)\right|=\infty$.

Preuve. Evident en utilisant la définition de la conjugaison. 
LEMme 2. Soit $\left(U_{n}\right)_{n \in \mathbb{N}}$ une suite strictement croissante d'entiers positifs définie à partir d'une relation de récurrence de la forme

$$
U_{n+m+1}=a_{0} U_{n+m}+\ldots+a_{m-1} U_{n+1}+a_{m} U_{n}
$$

avec $a_{0}, \ldots, a_{m-1}$ des entiers relatifs, $a_{m}$ égal à \pm 1 et $U_{0}, \ldots, U_{m}$ des entiers naturels. Soit $k$ un entier positif strictement plus grand que 1. Considérons la suite infinie d'entiers $\left(U_{n, t}\right)_{n \in \mathbb{N}}$ appartenant $\grave{a}\left\{0, \ldots, k^{t}-1\right\}$ ( $t$ entier $>1)$, définie par la suite $\left(U_{n}\right)_{n \in \mathbb{N}}$ modulo $k^{t}$. Alors, la suite $\left(U_{n, t}\right)_{n \in \mathbb{N}}$ est périodique de période $T_{t}$ et $\lim _{t \rightarrow \infty} T_{t}=\infty$.

Preuve. Nous savons que $\left(U_{n, t}\right)_{n \in \mathbb{N}}$ est ultimement périodique car engendrée par une relation de récurrence linéaire $([11])$. Comme $\left(U_{n}\right)_{n \in \mathbb{N}}$ est une suite strictement croissante et que $a_{m}$ est égal à \pm 1 , il est facile de montrer que la suite $\left(U_{n, t}\right)_{n \in \mathbb{N}}$ est périodique pour $t$ entier $>1$.

Pour $t$ entier $>1$, on peut toujours trouver $s$ entier tel que $U_{T_{t}}<k^{s}$, et comme $\left(U_{n, s}\right)_{n \in \mathbb{N}}$ est une suite périodique, nous avons $T_{t}<T_{s}$. Nous en déduisons que $\lim _{t \rightarrow \infty} T_{t}=\infty$.

Preuve de la proposition 2 . Soit $\omega=\left(\omega, A, a_{0}\right)$ une substitution satisfaisant à toutes les hypothèses de la proposition. Nous allons supposer que $X_{\omega}$ est $\theta$-automatique : il existe donc une $\theta$-substitution $\sigma=\left(\sigma, B, b_{0}\right)$ et une application $\Phi$ de $B$ dans $A$ telles que $\Phi\left(X_{\sigma}\right)=X_{\omega}$.

Soit $Y \in B^{\mathbb{N}}$ avec $X_{\sigma}=b_{0} Y$. Soit l'application $f: \mathbb{N} \rightarrow B, n \mapsto$ $f(n)=b$ si $b$ est la première lettre de $\sigma^{n}(Y)$. L'application $f$ est ultimement périodique :

$$
\exists(p, r) \in \mathbb{N}^{2} \forall n \geq r, \quad f(n+p)=f(n) .
$$

Ceci implique que pour tout $n \geq r$ (avec $f(n)=b$ ), et pour tous $(i, j) \in \mathbb{N}^{2}$ tel que $j \geq i$, le mot $\sigma^{i p}(b)$ est un préfixe du mot $\sigma^{n+j p}(Y)$.

Considérons la suite $\left(U_{n}\right)_{n \in \mathbb{N}}$ base du $\theta$-système de numération. Grâce aux propriétés de la conjugaison nous avons $\left|\sigma^{n}\left(b_{0}\right)\right|=U_{n}$ pour tout $n \in \mathbb{N}$.

Fixons $t$ entier tel que la période (notée $T=T_{t}$ ) de la suite $\left(U_{n, t}\right)_{n \in \mathbb{N}}$ soit supérieure strictement à $p$ (notations du lemme 2) : cela est possible car la suite $\left(U_{n}\right)_{n \in \mathbb{N}}$ vérifie les hypothèses du lemme 2 . On peut donc trouver $n$ (avec $f(n)=b$ ) supérieur à $r$ tel que $U_{n}$ ne soit pas congru modulo $k^{t}$ à $U_{n+p}$. Mais alors, pour tout entier $i, U_{n+i p T}$ n'est pas congru modulo $k^{t}$ à $U_{n+p+i p T}$. Cependant d'après une remarque ci-dessus, pour tout entier $i$, le mot $\sigma^{i p T}(b)$ est préfixe du mot $\sigma^{n+i p T}(Y)$ et du mot $\sigma^{n+p+i p T}(Y)$.

Dans $X_{\sigma}$, nous avons donc trouvé une suite de mots $\left(\sigma^{i p T}(b)\right)_{i \in \mathbb{N}}$ tels que $\lim _{i \rightarrow \infty}\left|\sigma^{i p T}(b)\right|=\infty$ (lemme 1), et pour tout entier $i$, le mot $\sigma^{i p T}(b)$ apparaît dans $X_{\sigma}$ à des rangs non congrus modulo $k^{t}$. Comme par hypôthèse $\Phi\left(X_{\sigma}\right)=X_{\omega}$, en considérant la suite de mots $\left(\Phi\left[\sigma^{i p T}(b)\right]\right)_{i \in \mathbb{N}}$, nous obtenons une contradiction avec la reconnaissabilité à l'ordre $t$ de la substitution $\omega$. Ceci achève la preuve de la proposition 2 . 
2. Une classification des substitutions. Soient $\omega=\left(\omega, A, a_{0}\right)$ une substitution et $a$ une lettre de $A$; considérons le sous-ensemble $A(a)$ de $A$ suivant :

$$
A(a)=\left\{a^{\prime} \in A: \exists(i, j) \in \mathbb{N}^{*} \text { avec } a^{\prime} \in \omega^{i}(a) \text { et } a \in \omega^{j}\left(a^{\prime}\right)\right\} .
$$

Propriétés immédiates:

1. $A\left(a_{0}\right) \neq \emptyset\left(\operatorname{car} a_{0} \in A\left(a_{0}\right)\right)$.

2. $A(a)$ peut être vide même si $a$ est une lettre récurrente de $A$.

3. Si $a^{\prime} \in A(a)$ alors $A\left(a^{\prime}\right)=A(a)$.

4. Si $X_{\omega} \neq\left(a_{0}\right)^{\omega}$, il existe $a \neq a_{0}$ tel que $A(a) \neq \emptyset$.

5. Soit $a \neq a_{0}$ et $a \in A\left(a^{\prime}\right)$; alors $a$ est une lettre récurrente de $A$.

EXEMPle 2. Soit $\omega=(\omega,\{0,1, \ldots, 5\}, 0)$ la 2 -substitution suivante :

$$
\begin{array}{cll}
\omega: & 0 \rightarrow 01 & 3 \rightarrow 13 \\
& 1 \rightarrow 23 & 4 \rightarrow 54 \\
& 2 \rightarrow 45 & 5 \rightarrow 45 .
\end{array}
$$

Sur cet exemple nous avons : $A(0)=\{0\}, A(1)=A(3)=\{1,3\}, A(4)=$ $A(5)=\{4,5\}$. La lettre 2 est récurrente bien que l'ensemble $A(2)$ soit vide.

Nous pouvons classer les substitutions $\omega=\left(\omega, A, a_{0}\right)$ en trois catégories :

(a) Toutes les lettres de $A$ appartiennent à $A\left(a_{0}\right)$ (toutes les lettres sont alors récurrentes).

(b) Il existe au moins deux ensembles différents $A(a)$ et $A\left(a^{\prime}\right)$ non vides ne contenant que des lettres récurrentes.

(c) La lettre $a_{0}$ n'est pas récurrente, et toutes les lettres récurrentes appartiennent au même ensemble $A(a)$ (ce qui implique en particulier que si $a^{\prime} \neq a_{0}$ et $A\left(a^{\prime}\right) \neq \emptyset$, alors $\left.a^{\prime} \in A(a)\right)$.

Soit $\omega=\left(\omega, A, a_{0}\right)$ une substitution rentrant dans la catégorie (b) cidessus, et notons :

- $A_{1}, \ldots, A_{n}$ tous les sous-ensembles deux à deux distincts de la forme $A(a)$ de $A$;

- $a_{1}, \ldots, a_{m}$ les lettres récurrentes de $A$ n'appartenant à aucun $A_{i}, i \in$ $\{1, \ldots, n\}$

- $\Omega=\left\{A_{1}, \ldots, A_{n}\right\} \cup\left\{a_{1}, \ldots, a_{m}\right\}$.

Nous formons des chaînes $(x \rightarrow y \rightarrow \ldots \rightarrow z)$ d'éléments de $\Omega$ de la façon suivante :

- $A_{i} \rightarrow A_{j}$, pour $i$ différent de $j$, s'il existe $a \in A_{i}$ et $a^{\prime} \in A_{j}$ telles que $a^{\prime} \in \omega(a)$

- $A_{i} \rightarrow a_{j}$ s'il existe $a \in A_{i}$ telle que $a_{j} \in \omega(a)$;

- $a_{j} \rightarrow A_{i}$ s'il existe $a \in A_{i}$ telle que $a \in \omega\left(a_{j}\right)$;

- $a_{i} \rightarrow a_{j}$ si $a_{j} \in \omega\left(a_{i}\right)$. 
Il est alors impossible d'avoir simultanément $x \rightarrow y$ et $y \rightarrow x$ (pour $x$ et $y$ deux éléments différents de $\Omega$ ). De plus, nous interdisons $A_{i} \rightarrow A_{i}$. Nous obtenons :

Proposition 3. Il existe dans $\Omega$ des chaînes de longueur maximale (construites comme ci-dessus). L'extrémité finale de ces chaînes est un élément de $\left(A_{i}\right)_{i \in\{1, \ldots, n\}}$ (noté $\left.A_{\mathrm{f}}\right)$ qui vérifie :

$\forall a \in A_{\mathrm{f}}, \quad \omega(a)$ est un mot formé de lettres de $A_{\mathrm{f}}$.

Preuve. Comme un élément de $\Omega$ ne peut pas apparaître deux fois dans la même chaîne (car sinon nous obtenons une contradiction avec les définitions), et que le cardinal de $\Omega$ est fini, toutes les chaînes que nous pouvons construire sont de longueur finie. Considérons une chaîne de longueur maximale: $x \rightarrow y \rightarrow \ldots \rightarrow z$. Comme pour toute lettre $a$ de $z$ le mot $\omega(a)$ est défini, $z$ ne peut pas être un élément de $\left(a_{j}\right)_{j \in\{1, \ldots, m\}}$, et doit contenir toutes les lettres de $\omega(a)$.

EXEMPle 3. Reprenons la substitution $\omega=(\omega,\{0,1, \ldots, 5\}, 0)$ de l'exemple 2. Alors $\Omega=\{A(0), A(1), A(4), 2\}$. Il y a une chaîne de longueur quatre: $A(0) \rightarrow A(1) \rightarrow 2 \rightarrow A(4)$. L'ensemble $A(4)$ vérifie bien que pour tout $a \in A(4), \omega(a)$ est un mot formé de lettres de $A(4)$.

\section{Un théorème}

THÉORÈme 1. Soient $\theta$ un nombre de Pisot unitaire ayant la propriété $(\mu), k$ un entier strictement plus grand que 1 et $\omega=\left(\omega, A, a_{0}\right)$ une $k$ substitution non ultimement périodique; alors $X_{\omega}$ n'est pas une suite $\theta$ automatique.

Pre u ve. Nous allons démontrer le théorème selon l'appartenance de la substitution $\omega$ à l'une des trois catégories (a)-(c) définies au paragraphe B.2 :

(a) La substitution $\omega$ est primitive, elle est alors reconnaissable à tous les ordres ([8]). Le mot $X_{\omega}$ n'est pas une suite $\theta$-automatique (proposition 2).

(b) Avec les notations de la proposition 3, soit $A_{\mathrm{f}}$ l'extrémité finale d'une chaîne de longueur maximale d'éléments de $\Omega$. Nous construisons la $k$-substitution non ultimement périodique $\tau=\left(\tau,\left(A \backslash A_{\mathrm{f}}\right) \cup\{b\}, a_{0}\right)$ (où $b$ n'est pas une lettre de $A$ ) suivante :

- pour $a \in A \backslash A_{\mathrm{f}}, \quad \tau(a)$ est le mot de $\left(\left(A \backslash A_{\mathrm{f}}\right) \cup\{b\}\right)^{*}$ obtenu en remplaçant dans $\omega(a)$ toutes les lettres de $A_{\mathrm{f}}$ par $b$;

- $\tau(b)$ est le mot $b^{k}$.

Il existe un morphisme $h$ (§A.2) de $\tau$ dans $\omega$ défini par

$$
h: A \rightarrow\left(A \backslash A_{\mathrm{f}}\right) \cup\{b\}, \quad a \mapsto h(a)= \begin{cases}a & \text { si } a \in A \backslash A_{\mathrm{f}}, \\ b & \text { si } a \in A_{\mathrm{f}} .\end{cases}
$$


Comme le mot $X_{\tau}$ ne peut pas être une suite $\theta$-automatique (proposition 1 ), $X_{\omega}$ ne l'est donc pas.

(c) Si la matrice d'occurrences de la substitution $\omega$ est apériodique, alors $\omega$ est reconnaissable à tous les ordres ([8]) et donc $X_{\omega}$ n'est pas une suite $\theta$-automatique (proposition 2).

Si la matrice d'occurrences de la substitution $\omega$ est périodique, de période $d$, alors $\omega^{d}$ est une substitution de la catégorie (b) ci-dessus, et comme $X_{\omega}=X_{\omega^{d}}, X_{\omega}$ n'est pas une suite $\theta$-automatique.

Toutes les $k$-substitutions non ultimement périodiques appartiennent à l'une des trois catégories ci-dessus, le théorème en découle.

\section{Une généralisation du théorème de Cobham}

1. Une propriété des ensembles $U_{\theta}$-reconnaissables

Proposition 4. Soient $\theta$ un nombre de Pisot unitaire ayant la propriété $(\mu)$, et $S$ une partie infinie $U_{\theta}$-reconnaissable de $\mathbb{N}$. Pour deux entiers quelconques a et $b$, nous définissons l'ensemble $S(a, b)=\{n \in \mathbb{N}: a n+b \in S\}$. Alors $S(a, b)$ est $U_{\theta}$-reconnaissable.

Preuve. Nous ne donnons que la démarche utilisée pour démontrer le résultat. La proposition est équivalente à dire que pour toute suite $\left(x_{n}\right)_{n \in \mathbb{N}}$ $\theta$-automatique, les suites extraites $\left(x_{a n+b}\right)_{n \in \mathbb{N}}(a, b$ entiers $)$ sont $\theta$-automatiques. Ceci se montre en deux étapes :

- on montre que les suites extraites $\left(x_{n+b}\right)_{n \in \mathbb{N}}$ sont $\theta$-automatiques,

- on montre que les suites extraites $\left(x_{a n}\right)_{n \in \mathbb{N}}$ sont $\theta$-automatiques.

La deuxième étape est la partie technique de la démonstration.

\section{Une généralisation du théorème de Cobham}

Proposition 5. Soient $\theta$ un nombre de Pisot unitaire ayant la propriété $(\mu), k$ un entier strictement plus grand que 1 et $S$ une partie infinie de $\mathbb{N}$ $k$-reconnaissable et $U_{\theta}$-reconnaissable. Si $\Delta_{k}=\left\{Q, q_{0}, F,\{0, \ldots, k-1\}, \delta\right\}$ est le $k$-automate minimal complet reconnaissant $S$, alors, quel que soit l'état $q$ de $\Delta_{k}$, l'ensemble $S(q)=\left\{n \in \mathbb{N}: \delta\left(q_{0}, \nu_{k}(n)\right)=q\right\}$ est $k$-reconnaissable et $U_{\theta}$-reconnaissable.

Preuve. En utilisant le résultat de la proposition 4, la preuve est identique à celle du lemme 1 de l'article de Cobham ([2]).

Corollaire 1. Avec les hypothèses et les notations de la proposition 5, la suite infinie des états $\left(q_{n}\right)_{n \in \mathbb{N}}\left(=\left[\delta\left(q_{0}, \nu_{k}(n)\right)\right]_{n \in \mathbb{N}}\right)$ de $\Delta_{k}$ est $\theta$-automatique.

Preuve. Supposons que $\Delta_{k}$ se compose de $i+1$ états $q_{0}, q_{1}, \ldots, q_{i}$. Pour chaque état $q_{j}$, nous considérons le $\theta$-automate $\Delta_{\theta}(j)$ reconnaissant 
l'ensemble $S\left(q_{j}\right)$ (proposition 5). Nous construisons alors le $\theta$-automate $\Delta_{\theta}$, produit des $\theta$-automates $\Delta_{\theta}(j)$. Chaque état de $\Delta_{\theta}$ est un $(i+1)$-uplé $\left(p_{0}, p_{1}, \ldots, p_{i}\right)$ où $p_{j}$ est un état de $\Delta_{\theta}(j)$. Ces $(i+1)$-uplés comportent un seul état $p_{j}$ final dans $\Delta_{\theta}(j)$ (sinon contradiction avec la définition des automates $\left.\Delta_{\theta}(j)\right)$.

Nous considérons l'application $\Phi$ définie par $\Phi\left(\left(p_{0}, p_{1}, \ldots, p_{i}\right)\right)=q_{j}$ si $p_{j}$ est final dans $\Delta_{\theta}(j)$. Si $\left(\left(p_{0}, p_{1}, \ldots, p_{i}\right)_{n}\right)_{n \in \mathbb{N}}$ désigne la suite des états de $\Delta_{\theta}$, nous avons alors $\Phi\left[\left(\left(p_{0}, p_{1}, \ldots, p_{i}\right)_{n}\right)_{n \in \mathbb{N}}\right]=\left(q_{n}\right)_{n \in \mathbb{N}}$. La suite infinie des états de $\Delta_{k}$ est donc $\theta$-automatique.

ThÉORÈme 2. Soient $\theta$ un nombre de Pisot unitaire ayant la propriété $(\mu)$ et $k$ un entier strictement plus grand que 1 . Une partie infinie de $\mathbb{N}$ est $k$-reconnaissable et $U_{\theta}$-reconnaissable si et seulement si elle est ultimement périodique.

Preuve. Nous savons que les sous-ensembles ultimement périodiques de $\mathbb{N}$ sont $U_{\theta}$-reconnaissables ([6]), et $k$-reconnaissables (propriété ancienne, voir [4] par exemple).

Si $S$ est une partie infinie, $k$-reconnaissable et $U_{\theta}$-reconnaissable de $\mathbb{N}$, alors la suite infinie des états du $k$-automate minimal, complet, reconnaissant $S$ est $\theta$-automatique (corollaire 1 ). Cependant, cette suite peut être considérée comme le point fixe d'une $k$-substitution ([3]); elle est donc ultimement périodique (théorème 1 ), et $S$ est une partie ultimement périodique de $\mathbb{N}$.

Remarques générales. Une démarche analogue peut être utilisée pour démontrer le théorème original de Cobham avec deux entiers $k$ et $j$ premiers entre eux (voir $\S A$ ).

On peut aussi appliquer la même démarche, et obtenir un théorème analogue, en considérant un nombre de Pisot $\theta$ (pas forcément unitaire) ayant la propriété $(\mu)$, et un entier $k(>1)$ premier avec le coefficient constant du polynôme minimal de $\theta$.

Remerciements. Je remercie les professeurs G. Rauzy et B. Host pour leurs conseils et leurs encouragements qui m'ont permis de mener à bien ce travail.

\section{Bibliographie}

[1] A. Bertrand, Répartition modulo 1 des suites exponentielles et systèmes dynamiques symboliques, Thèse d'état, Université de Bordeaux 1, 1986.

[2] A. Cobham, On the base dependence of sets of numbers recognizable by finite automata, Math. Systems Theory 3 (1969), 186-192.

[3] —, Uniform tag sequence, ibid. 6 (1972), 164-192.

[4] S. Eilenberg, Automata, Languages and Machines, Vol. A, Academic Press, 1974. 
[5] S. Fabre, Substitutions et $\beta$-systèmes de numération, preprint.

[6] C. Frougny, Systèmes de numération linéaires et automates finis, Thèse d'état, Université Paris-VII, 1989.

[7] G. Hansel, A propos d'un théorème de Cobham, Acte de la fête des mots, D. Perrin (ed.), Rouen, 1982, Greco de programmation, C.N.R.S.

[8] B. Mossé, Puissances de mots et reconnaissabilité des points fixes d'une substitution, Theor. Comput. Sci. 99 (1992), 327-334.

[9] W. Parry, On the $\beta$-expansions of real numbers, Acta Math. Acad. Sci. Hungar. 11 (1960), 401-416.

[10] M. Queffélec, Substitution Dynamical Systems. Spectral Analysis, Lecture Notes in Math. 1294, Springer, Berlin, 1987.

[11] G. Rauzy, Relations de récurrence modulo $m$, Séminaire Delange-Pisot, 5ième année (1963-1964), (2-01)-(2-10).

[12] E. Zeckendorf, Représentation des nombres naturels par une somme de nombres de Fibonacci ou de nombres de Lucas, Bull. Soc. Roy. Sci. Lièges 3-4 (1960), $179-182$.

UNIVERSITÉ PARIS XIII

DÉPARTEMENT DE MATHÉMATIQUES

AVENUE J.-B. CLÉMENT

93430 VILLETANEUSE, FRANCE 